\title{
On the heterophase liquid thermodynamics and cooperative dynamics
}

\author{
O.S. Bakai \\ National Scientific Center "Kharkiv Institute of Physics and Technology", \\ 1 Akademicheskaya Str., 61108, Kharkiv, Ukraine
}

Received February 22, 2010

The thermodynamics and cooperative dynamics of heterophase liquid states is considered taking into account frustration and volumetric interaction of the solid-like fluctuons. It is found that the glass transition temperature range is scaled by difference of the frustration parameter and mean energy of the volumetric interaction. A model of the cooperative relaxation with finite cooperatively rearranging domains is considered. The fictitious Kauzmann and Vogel-Fulcher temperatures are determined. It is found that they are close to a proper accuracy. Correlation of the temperatures of glass transition, Vogel-Fulcher and "ideal" glass transition (as it is determined in the mode coupling model) is considered too.

Key words: heterophase liquid states, glass transition, Kauzmann and Vogel-Fulcher temperatures

PACS: $64.70 . J a, 64.70 . P f, 61.20 . G y$

\section{Introduction}

The heterophase fluctuations model (HPFM) is based on the idea of heterophase structure of glass forming liquids [1 [5]. Consecutive consideration shows that in a heterophase state (HPS), molecular clusters (fluctuons) of specified short-range order (SRO) are statistically significant entities rather than single molecules. As a result, the supramolecular interactions determine the thermodynamics and dynamics of the glass forming liquids. Equation for the free energy of HPS is deduced within the framework of HPFM taking into account frustration and volumetric interaction of the fluctuons [5, 6]. The model of the cooperative relaxation dynamics of HPS developed in [2, 3] describes the $\alpha$-relaxation rate in terms of the free energy. The developed theory is a proper base for the analysis of experimental data on thermodynamics, structure and cooperative relaxation of the glass-forming liquids 2,4$]$.

The Vogel-Fulcher and Kauzmann temperatures $\left(T_{\mathrm{VF}}\right.$ and $\left.T_{\mathrm{K}}\right)$ [9] and the temperature of the "ideal" glass transition determined within the framework of the mode-coupling model $\left(T_{\mathrm{MCT}}\right)$ [10, 11] are in use at the analysis and rationalization of experimental data. At $T_{\mathrm{K}}$ the extrapolated entropy of liquid becomes equal to that of crystal or, in other words, the configurational entropy of liquid is going to zero. In [9, 12] it is assumed that a critical point is located at Kauzmann temperature, $T_{\mathrm{K}}<T_{\mathrm{g}}$ while $T_{\mathrm{VF}}$ is the assumed singular point of the cooperative relaxation [7, 8]. The relaxation time at this point is $\sim \exp \left(T_{\mathrm{K}} /\left(T-T_{\mathrm{K}}\right)\right)$. The empiric Vogel-Fulcher formula was obtained by Adam and Gibbs in a theoretical model [13]. They connected the size of the cooperatively rearranging domain with the configurational entropy of liquid. The size increases with the configurational entropy decrease while at $T_{\mathrm{K}}$ it goes to infinity. As a result, $T_{\mathrm{K}}$ is a singular point of the cooperative relaxation and $T_{\mathrm{K}}=T_{\mathrm{VF}}$. Though $T_{\mathrm{K}}$ and $T_{\mathrm{VF}}$ are not accessible for a direct observation, the formulas obtained in Gibbs-DiMarzio and Adam-Gibbs (AG) models (along with the empiric definition by Kauzmann) make it possible to rationalize and fit the experimental data on the liquid configurational entropy and relaxation cooperative rates.

Determination of $T_{\mathrm{MCT}}$ is given in terms of the basic relations of thermodynamic and dynamic properties of glass-forming liquids under special assumptions [10, 11]. The predicted "ideal" glass transition has not been observed yet but this model and its consequences have been under consid- 
eration for several decades. In Odagaki's model of the cooperative relaxation [14] and in [15], where this model is reconsidered without resource of the configurational entropy arguments, the following correlations of the singular temperatures are found: $T_{\mathrm{K}}=T_{\mathrm{VF}}$ and $T_{\mathrm{MCT}}-T_{\mathrm{VF}}=2\left(T_{\mathrm{g}}-T_{\mathrm{VF}}\right)$.

By means of experimental data fittings, the mentioned temperatures are determined for many glass-forming liquids. It is found that $T_{\mathrm{MCT}}>T_{\mathrm{g}}$ and correlations $T_{\mathrm{K}} \approx T_{\mathrm{VF}}$ and $T_{\mathrm{MCT}}-T_{\mathrm{VF}} \approx$ $2\left(T_{\mathrm{g}}-T_{\mathrm{VF}}\right)$ are true with an acceptable accuracy (see e.g. [15 17]). For this reason, it is desirable to check whether these correlations are consistent with the HPFM predictions. This consideration is performed below. Furthermore, some peculiarities of the HPFM including volumetric interaction and frustration of solid-like fluctuons are considered.

In section 2, the thermodynamics of HPS is considered. Section 3 is devoted to a description of cooperative relaxation dynamics in terms of thermodynamic properties of glass-forming liquid. Consideration of the mentioned correlations and discussion is placed in section 4 .

\section{Glass transition in HPFM model}

To get the characteristic temperatures $T_{\mathrm{K}}$ and $T_{\mathrm{VF}}$, whose definitions have no connection with microstructure of glass-forming liquid, the coarsened HPFM without specification of the short range order of solid-like fluctuons has to be used. In this formulation of the HPFM [1, 3, [6], solidlike fluctuons having the averaged SRO and thermodynamic properties are considered. In this case, the order parameters of the liquid are fractions of the solid-like and fluid-like fluctuons, $c_{\mathrm{s}}$ and $c_{\mathrm{f}}$, respectively. Due to the evident relation,

$$
c_{\mathrm{s}}+c_{\mathrm{f}}=1
$$

only one of these parameters is independent.

For simplification, the number of molecules per the solid-like and fluid-like fluctuon are put to be equal, $k_{\mathrm{s}}=k_{\mathrm{f}}=k_{0}$. In this case the free energy in the mean-field approximation is

$$
G(P, T)=\int g(x ; P, T) \mathrm{d}^{3} x .
$$

Its density can be presented as follows:

$$
\begin{aligned}
g(x, P, T)= & c_{\mathrm{f}}(x) g_{\mathrm{f}}^{0}+c_{\mathrm{s}}(x) g_{\mathrm{s}}^{0}+c_{\mathrm{s}}(x) c_{\mathrm{f}}(x) g_{\mathrm{sf}}+\frac{1}{2} g_{\mathrm{ss}} c_{\mathrm{s}}^{2} \\
& +\frac{1}{2} \int c_{\mathrm{s}}(x) \Phi\left(x, x^{\prime}\right) c_{\mathrm{s}}\left(x^{\prime}\right) \mathrm{d}^{3} x^{\prime}+T\left(c_{\mathrm{f}} \ln c_{\mathrm{f}}+c_{\mathrm{s}} \ln c_{\mathrm{s}}\right) .
\end{aligned}
$$

Here $g_{i}^{0}(P, T)$ is the part of free energy of $i$-th fluctuon independent of fractions $\left\{c_{k}\right\} ; g_{\mathrm{sf}}^{0}(P, T)>0$ is the interfacial energy; $g_{\mathrm{ss}}>0$ is the frustration parameter; $\Phi\left(x, x^{\prime}\right)$ is the attractive potential of the solid-like fluctuons which we take in the Yukawa form

$$
\Phi\left(x, x^{\prime}\right)=-\frac{r_{0} \varphi}{r} \exp \left(-r / R_{0}\right)
$$

where $r_{0}$ is the fluctuon size; $\varphi>0$ and $R_{0}>r_{0}$ is the strength and range of the potential, respectively. The last term in (3) describes the contribution of the mixing entropy of solid-like and fluid-like fluctuons.

The attractive volumetric interaction of fluctuons can lead to long-range correlations of solidlike fluctuons (the Fischer cluster formation) [4, [6]. The contribution of long-range correlations of solid-like fluctuons into the free energy is comparatively small [6] and can be neglected for a while. Therefore, we can replace $c_{\mathrm{S}}(x)$ by its mean value $c_{\mathrm{s}}$ in (3). As result, equation (3) reads

$$
\begin{gathered}
g(x, P, T) \Rightarrow g(P, T)=c_{\mathrm{f}} g_{\mathrm{f}}^{0}+c_{\mathrm{s}} g_{\mathrm{s}}^{0}+c_{\mathrm{s}} c_{\mathrm{f}} g_{\mathrm{sf}}+\frac{1}{2}\left(g_{\mathrm{ss}}-\varphi_{0}\right) c_{\mathrm{s}}^{2}+T\left(c_{\mathrm{f}} \ln c_{\mathrm{f}}+c_{\mathrm{s}} \ln c_{\mathrm{s}}\right) \\
\varphi_{0}=4 \pi \int \Phi(r) r^{2} \mathrm{~d} r^{\prime}=-4 \pi R_{0}^{2} \varphi
\end{gathered}
$$


The equation determining the liquid equilibrium state, $\partial g / \partial c_{\mathrm{s}}=0$, reads

$$
\begin{gathered}
\left(1-2 c_{\mathrm{s}}\right) \tilde{g}_{\mathrm{sf}}+T \ln \frac{c_{\mathrm{s}}}{1-c_{\mathrm{s}}}=h_{\mathrm{sf}}, \\
\tilde{g}_{\mathrm{sf}}=g_{\mathrm{sf}}-\left(g_{\mathrm{ss}}-\varphi_{0}\right) / 2, \quad h_{\mathrm{sf}}=g_{\mathrm{f}}^{0}-g_{\mathrm{s}}^{0}-\left(g_{\mathrm{ss}}-\varphi_{0}\right) / 2 .
\end{gathered}
$$

It follows from (77) that the phase coexistence temperature, $T_{\mathrm{e}}$, (at which the solution $c_{\mathrm{S}}=1 / 2$ exists) is determined by the following equation

$$
h_{\mathrm{sf}}=0 .
$$

To show the effect of the frustration and volumetric interaction on the equilibrium and glass transition, let us introduce the phase equilibrium temperature without fluctuon interactions as follows:

$$
T_{\mathrm{e}}^{0}=\Delta \varepsilon_{\mathrm{sf}} / \Delta s_{\mathrm{sf}}
$$

Here $\Delta \varepsilon_{\mathrm{sf}}$ and $\Delta s_{\mathrm{sf}}$ is the difference of energies and entropies of the non-interacting solid-like and fluid-like fluctuons at the point where $g_{\mathrm{s}}^{0}(P, T)=g_{\mathrm{f}}^{0}(P, T)$. Then, as it follows from (8), the phase coexistence temperature is,

$$
T_{\mathrm{e}}=T_{\mathrm{e}}^{0}-\left(g_{\mathrm{ss}}-\varphi_{0}\right) / 2 \Delta s_{\mathrm{f}, \mathrm{s}} .
$$

The frustration decreases while the volumetric interaction increases the phase coexistence temperature.

Apparently, for the majority of substances the glass transition proved to be continuous. It follows from equation (7) that the continuous transformation takes place if

$$
\tilde{g}_{\mathrm{sf}} \leqslant 2 T_{\mathrm{e}}
$$

or

$$
g_{\mathrm{sf}}<2\left[T_{\mathrm{e}}^{0}+\left(g_{\mathrm{ss}}-\varphi_{0}\right)\left(1-1 / \Delta s_{\mathrm{f}, \mathrm{s}}\right) / 2\right]
$$

Taking into account that the difference of entropies of fluid and solid is $\sim 1$ per molecule and that $k_{0} \sim 10$, we have $\Delta s_{\mathrm{f}, \mathrm{s}} \gg 1$. Therefore, with $g_{\mathrm{ss}} \sim g_{\mathrm{sf}}$ the condition (10) can be easily fulfilled.

It is of particular interest to consider the heterophase fluctuations in Frenkel's limit [18], at $c_{\mathrm{s}} \rightarrow 1$ and $c_{\mathrm{s}} \rightarrow 0$, i.e., for almost pure solid and fluid phases with small amount of heterophase fluctuations. In these cases, the solutions of equation (77) take the following form

$$
\begin{aligned}
& c_{\mathrm{s}}^{(2)}(T)=1-\exp \left\{\left[\Delta s_{\mathrm{f}, \mathrm{s}}\left(T-T_{\mathrm{e}}^{1}\right)-g_{\mathrm{sf}}\right] / T_{\mathrm{e}}^{1}\right\} \\
& c_{\mathrm{s}}^{(3)}(T)=\exp \left\{\left[\Delta s_{\mathrm{f}, \mathrm{s}}\left(T_{\mathrm{e}}^{0}-T\right)-g_{\mathrm{sf}}\right] / T_{\mathrm{e}}^{0}\right\}
\end{aligned}
$$

where

$$
T_{\mathrm{e}}^{1}=T_{\mathrm{e}}^{0}-g_{\mathrm{ss}} / \Delta s_{\mathrm{f}, \mathrm{s}}=T_{\mathrm{e}}^{0}-\Delta T_{\mathrm{g}} .
$$

Evidently, $\Delta T_{\mathrm{g}}=g_{\mathrm{ss}} / \Delta s_{\mathrm{f}, \mathrm{s}}$ is the glass transition temperature range of the equilibrium liquid. We remind that the glass transition temperature $T_{\mathrm{g}}$ depends on the cooling rate because in the vicinity of $T_{\mathrm{e}}^{1}$ the relaxation dynamics dramatically slows down, the liquid becomes non-equilibrium and non-ergodic. Therefore, calorimetrically determined $T_{\mathrm{g}}$ is above $T_{\mathrm{e}}^{1}$.

From equations (13)-(15) we see that the frustration, apart from decreasing the phase coexistence temperature $T_{\mathrm{e}}$, causes the appearance of two characteristic temperatures, $T_{\mathrm{e}}^{0}$ and $T_{\mathrm{e}}^{1}$. The physical meaning of the temperatures $T_{\mathrm{e}}^{0}$ and $T_{\mathrm{e}}^{1}$ is clear. At $T_{\mathrm{e}}^{0}$, the transformation of an almost homogeneous fluid into the heterophase liquid state takes place. The non-Frenkelian heterophase liquid state exists within the temperature range $\left[T_{\mathrm{e}}^{0}, T_{\mathrm{e}}^{1}\right]$. The fraction $c_{\mathrm{s}}$ increases with the temperature decrease and at $T_{\mathrm{e}}^{1}$ the heterophase liquid transforms into a glass with weak fluidlike heterophase fluctuations. Therefore, $T_{\mathrm{e}}^{1}$ may be regarded as thermodynamic glass transition temperature. 
To complete the description of $c_{\mathrm{S}}(T)$ we find a solution of equation (6) in the vicinity of $T_{\mathrm{e}}$. It can be presented in the form of a series in $T-T_{\mathrm{e}}[1]$

$$
c_{\mathrm{s}}(T)=\frac{1}{2}+\frac{h_{\mathrm{s}, \mathrm{f}}(T)}{2\left(2 T-\tilde{g}_{\mathrm{s}, \mathrm{f}}\right)}\left[1-\frac{2 T_{\mathrm{e}} h_{\mathrm{s}, \mathrm{f}}^{2}(T)}{3\left(2 T-\tilde{g}_{\mathrm{s}, \mathrm{f}}\right)^{3}}\right]=\frac{1}{2}+\frac{T_{\mathrm{e}}-T}{2 \delta T}\left[1-\frac{2 T_{\mathrm{e}}\left(T_{\mathrm{e}}-T\right)^{2}}{3(\delta T)^{3}}\right],
$$

where

$$
\delta T=\frac{2 T-\tilde{g}_{\mathrm{s}, \mathrm{f}}}{\Delta s_{\mathrm{s}, \mathrm{f}}}
$$

To estimate the role of correlation of the solid like fluctuons, let us take the pair correlation function

$$
\begin{gathered}
w_{\mathrm{ss}}(r)=\left\langle c_{\mathrm{s}}(x) c_{\mathrm{s}}\left(x^{\prime}\right)\right\rangle=\bar{c}_{S} \omega_{\mathrm{ss}}(r)+\bar{c}_{\mathrm{s}}^{2}=\bar{c}_{\mathrm{s}}\left(\frac{r_{0}}{r}\right)^{3-D} \exp (-r / \xi)+\bar{c}_{\mathrm{s}}^{2}(1-\exp (-r / \xi)) \\
r \geqslant r_{0}, \quad r=\left|x-x^{\prime}\right|
\end{gathered}
$$

where $D \leqslant 3$ and $\xi \geqslant R_{0}$ is the fractal dimension and correlation length, respectively. The last term in (18) describes the input from non-correlated fluctuons. The exponent $\exp (-r / \xi)$ describes a smooth decrease of the pair correlation on the scale $r \sim \xi$. It follows from (18) that

$$
\omega_{\mathrm{ss}}=\left[\left(\frac{r_{0}}{r}\right)^{3-D}-\bar{c}_{\mathrm{s}}\right] \exp (-r / \xi), \quad r \geqslant r_{0} .
$$

Neglecting the long-range correlations, we can take for estimations $\xi \sim R_{0}$. Then the correlation energy is [].

$$
\begin{aligned}
\varepsilon_{\mathrm{corr}}\left(\bar{c}_{\mathrm{s}} ; D, \xi\right) & \approx 2 \pi \int_{r_{0}}^{\infty} \Phi(r) \omega_{\mathrm{ss}}(r) r^{2} \mathrm{~d} r^{\prime} \sim-\bar{c}_{\mathrm{s}}^{2}\left(\frac{\xi}{R_{0}+\xi}\right)^{D-1}\left|\varphi_{0}\right| \sim-\bar{c}_{\mathrm{s}}^{2}\left(1-(D-1) \frac{R_{0}}{\xi}\right)\left|\varphi_{0}\right| \\
\xi\left(\bar{c}_{\mathrm{s}}, D\right) & \approx\left(\bar{c}_{\mathrm{s}}\right)^{\frac{1}{D-3}} r_{0} .
\end{aligned}
$$

One can see from this equation that $\varepsilon_{\text {corr }}$ is proportional to $\bar{c}_{\mathrm{s}}^{2}, \varphi_{0}$. It increases with the temperature decrease due to an increase of $D, \xi$ and $\bar{c}_{\mathrm{s}}^{2}$. Formally, the correlation of the solid-like fluctuons can be taken into account by substitution $\varphi_{0} \rightarrow \tilde{\varphi}_{0}(T) \approx \varphi_{0}\left(1+\beta\left(T_{\mathrm{e}}-T\right)\right)$ in equations (7), (13) -(17). Here $\beta$ is the logarithmic derivative of $\tilde{\varphi}_{0}(T)$ at $T_{\mathrm{e}}$.

\section{The cooperative relaxational dynamics of the heterophase liquid $(\alpha$-relaxation)}

A model of the cooperative relaxational dynamics of the heterophase liquids was proposed in [2, 3]. It is based on a simple physical idea. The orientation and location of fluctuons are correlated due to the volumetric interaction on a length $\xi$ which is comparable to the interaction potential range $R_{0}$. Single jumps of molecules do not change the correlations within the correlated domain (CD). However, cooperative rearrangement, involving many molecules within CD can destroy and change the local correlation. Let us denote the size of the cooperatively rearranging domain (CRD) by $R_{\mathrm{CRD}}$. Evidently the cooperative rearrangement is an activated process. The number of the fluctuons involved in the rearrangement $z_{\mathrm{C}}$ is $\sim\left(R_{\mathrm{CRD}} / r_{0}\right)^{3}$ and the number of molecules within $\mathrm{CRD}$ is $\sim z_{\mathrm{C}} k_{0}$. To get an idea on the activation free energy let us consider the coarse-grained on the scale $r \sim R_{\mathrm{CRD}}$ free energy landscape of CRD's. It looks like this is shown in figure 1, The deepest minima of $g_{\mathrm{CRD}}$ belong to states with $c_{\mathrm{s}}(x)$ nearly equal to an equilibrium value, $\bar{c}_{\mathrm{s}}$, found in the previous section. The maxima of $g_{\mathrm{CRD}}$ belong to states with a small amount of solid-like fluctuons, which are responsible for the correlation settling, $c_{\mathrm{S}}(x) \ll 1$. In accordance with this picture we can determine the activation free energy of $\alpha$-relaxation at constant pressure as follows:

$$
g_{\mathrm{ac}}(T)=E+z_{\mathrm{C}}\left[g_{\mathrm{f}}^{0}(T)-g(T)+z_{\mathrm{C}}^{-1 / 3} c_{\mathrm{s}}(T) g_{\mathrm{sf}}\right]
$$




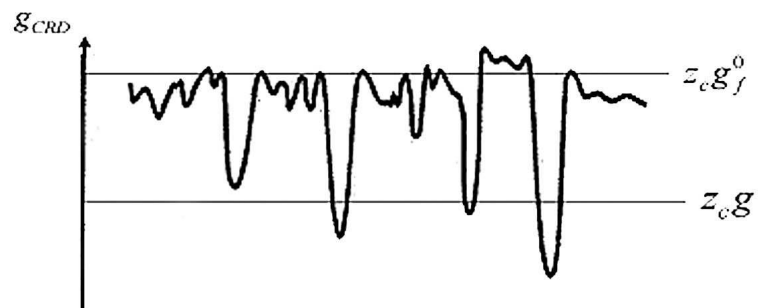

Configurational coordinate

Figure 1. Schematic pattern of the coarse-grained free energy landscape of the heterophase liquid. The free energy of CRD, $g_{\mathrm{CRD}}$, vs a configurational coordinate is shown. $z_{\mathrm{C}} g_{\mathrm{f}}^{0}$ is the free energy of "molten" CRD; $z_{\mathrm{C}} g$ is the equilibrium free energy of CRD.

$E$ is the activation energy of CRD in the fluid-like state. The term $\sim z^{2 / 3}$ is the interfacial free energy.

The cooperative relaxation plays a key role in the heterophase liquid existing at $T<T_{\mathrm{e}}^{0}$. The relaxation rate is

$$
\Gamma_{\alpha}(T)=\Gamma_{\alpha}^{0} \exp \left[-g_{\mathrm{ac}}(T) \beta\right],
$$

$\Gamma_{\alpha}^{0}=\Gamma\left(T_{\mathrm{e}}^{0}\right)$ is a constant; $\beta=1 / T$. The Boltzmann constant is put equal to 1 .

Considering $\Gamma_{\alpha}(T)$ in the vicinities of the characteristic temperatures $T_{\mathrm{e}}^{0}, T_{\mathrm{e}}^{1}$ and $T_{\mathrm{e}}$ where dependence of $c_{\mathrm{s}}$ on $T$ is given by expressions (13)-(17). At $\left|T-T_{\mathrm{e}}^{1}\right| \ll T_{\mathrm{e}}^{1}$ :

$$
\Gamma_{\alpha}(T)=\Gamma_{\alpha 1}=\Gamma_{\alpha}^{0} \exp \left(-\frac{E}{T}-c_{\mathrm{s}} \frac{z_{\mathrm{C}} \Delta s_{\mathrm{f}, \mathrm{s}}+z_{\mathrm{C}}^{2 / 3} g_{\mathrm{sf}}}{T}\right) .
$$

At $\left|T-T_{\mathrm{e}}\right| \ll T_{\mathrm{e}}$

$$
\begin{aligned}
& \left.\Gamma_{\alpha}(T)=\Gamma_{\alpha}^{(} T_{\mathrm{e}}\right) \exp \left\{-\frac{E}{T}-\frac{z_{\mathrm{C}} \Delta s_{\mathrm{f}, \mathrm{s}}}{2}\left[1+\frac{z_{\mathrm{C}}^{-1 / 3} g_{\mathrm{sf}}}{2 \Delta s_{\mathrm{f}, \mathrm{s}}\left(2 T_{\mathrm{e}}-\tilde{g}_{\mathrm{sf}}\right)}\right] \frac{T-T_{\mathrm{e}}}{T}\right\}, \\
& \Gamma_{\alpha}\left(T_{\mathrm{e}}\right)=\Gamma_{\alpha}^{0} 2^{-z_{\mathrm{C}}} \exp \left(-z_{\mathrm{C}} \frac{-\tilde{g}_{\mathrm{sf}}+z_{\mathrm{C}}^{-1 / 3} g_{\mathrm{sf}}}{T_{\mathrm{e}}}\right) .
\end{aligned}
$$

At $\left|T-T_{\mathrm{e}}^{0}\right| \ll T_{\mathrm{e}}^{0}:$

$$
\Gamma_{\alpha}(T)=\Gamma_{\alpha 0}(T)=\Gamma_{\alpha}^{0} \exp \left[z_{\mathrm{C}} \Delta s \frac{T-T_{\mathrm{e}}^{0}}{T}\right] .
$$

In [2, 3] these equations were successfully used to fit the relaxation rate and its derivatives of salol in the glass transition temperature range. Reliability of the developed $\alpha$-relaxation model was checked by fitting the experimental data for many other liquids. These results have not been published yet.

\section{Correlations of characteristic temperatures and discussion}

Let us refer to the models of a heterophase state with and without accounting the frustration and volumetric interaction of fluctuons as Model 1 and Model 2 respectively. The models differ in the thermodynamic coefficients. The substitution

$$
g_{\mathrm{s}}^{0} \rightarrow \tilde{g}_{\mathrm{s}}^{0}=g_{\mathrm{s}}^{0}+\left(g_{\mathrm{ss}}-\varphi_{0}\right) / 2, \quad g_{\mathrm{s}, \mathrm{f}} \rightarrow \tilde{g}_{\mathrm{ss}}=g_{\mathrm{s}, \mathrm{f}}-\left(g_{\mathrm{ss}}-\varphi_{0}\right) / 2
$$

leads to the transformation Model $1 \rightarrow$ Model 2 .

Due to the revealed independence of mathematical formulation of the Models on details of the pair interaction of solid-like fluctuons, we can expect that they are applicable for a description 
of glass-forming liquids of many types. It has to be noted that a variety of SRO of the solid-like fluctuons in some cases has to be taken into account like in [5] where two types of the solid-like fluctuons were taken in consideration to describe the liquid-liquid $1^{\text {st }}$ order phase transition.

The Model 2 has evident advantages. It accounts for important components of the solid-like fluctuon interaction - i.e., the frustration and volumetric interaction. Hence, we have got the determination of the glass transition temperature range $\left[T_{\mathrm{e}}^{1}, T_{\mathrm{e}}^{0}\right]$.

Further, in Model 1 the condition $g_{\mathrm{sf}}<2 T_{\mathrm{e}}$ has to be fulfilled to explain and describe the continuous liquid-to-glass transformation. This condition looks like unphysical, since $g_{\mathrm{sf}}$ is comparable with liquid - crystalline fluctuon interfacial free energy, $g_{\mathrm{cr}, \mathrm{f}}$, which is apparently larger than $2 T_{m}\left(T_{m}\right.$ is crystallization temperature) because crystallization is a discontinuous $1^{\text {st }}$ order phase transition. Model 2 is free of this shortcoming.

Thus, the HPFM is general enough to be applied to the examination of correlations of characteristic temperatures $T_{\mathrm{VF}}, T_{\mathrm{K}}, T_{\mathrm{MCT}}$ and $T_{\mathrm{g}}$.

The found expressions for $g(x, P, T)$ and its derivatives show no singularities at all temperatures. The relaxation rate (18) also has no singularities when the condition (11) is fulfilled. However, we can determine the Kauzmann temperature, $T_{\mathrm{K}}$, by extrapolation of the configurational entropy of liquid below $T_{\mathrm{e}}$.

To determine $T_{\mathrm{VF}}$ we have to compare the found $\alpha$-relaxation rate with the Vogel-Fulcher formula.

Since we have used the model of cooperatively rearranging domains, our approach has to be compared with the AG model of the $\alpha$-relaxtion controlled by cooperative rearrangements. In AG model, the configurational entropy per molecule, $s_{\mathrm{C}}$, is taken as a controlling parameter of the glass transition. A domain consisting of $z$ molecules has $W\left(z, s_{\mathrm{C}}\right) \approx \exp \left(z s_{\mathrm{C}}\right)$ structure states of the lowest energy. Structure rearrangement of the domain is possible if it has at least two different structure states, $W\left(z, s_{\mathrm{C}}\right)>2$. This condition gives lower boundary of CRD, $z>\ln 2 / s_{\mathrm{C}}$. One can see that with $s_{\mathrm{C}} \rightarrow 0$ the size of CRD increases and the transformation time increases too,

$$
\tau_{\alpha} \sim \exp \left(\frac{z \Delta \mu}{T}\right)=\exp \left(\frac{\text { Constant } \Delta \mu}{T s_{\mathrm{C}}}\right) .
$$

Here $\Delta \mu$ is the conventional free energy barrier per molecule to rearrangement. The Constant is $\sim 1$.

Taking into account that for glass-forming liquids

$$
\frac{s_{\mathrm{C}}}{T}<\frac{\partial s_{\mathrm{C}}}{\partial T}
$$

we see that with linear extrapolation of $s_{\mathrm{C}}(T)$

$$
s_{\mathrm{C}}(T)=0 \quad \text { at } \quad T_{\mathrm{K}}=T_{\mathrm{g}}-\left.\frac{s_{\mathrm{C}}}{\partial s_{\mathrm{s}} / \partial T}\right|_{T>T_{\mathrm{g}}}>0 .
$$

At $T_{\mathrm{K}}$, as equation (28) shows, $\tau_{\alpha} \rightarrow \infty$ as it should be at the $2^{\text {nd }}$ order phase transition.

In contrast to AG-model, we are considering CRDs with finite number of molecules, $z=z_{\mathrm{C}} \cdot k_{0}$. It was revealed experimentally [19 22] that in the vicinity of $T_{\mathrm{g}}$ the CRDs are finite. This property of cooperative relaxation was used to explain the dynamic heterogeneity of supercooled liquids [21, 23]. Due to this property $\tau_{\alpha}$ is finite below $T_{\mathrm{g}}$, as in the HPFM.

In a glass-forming liquid, the activation energy of rearrangement increases with the temperature decrease mainly due to the growth of $c_{\mathrm{s}}$ and supercooling, $g_{\mathrm{ac}} \sim z_{\mathrm{C}} \Delta s_{\mathrm{s}, \mathrm{f}}\left(T_{\mathrm{e}}-T\right) c_{\mathrm{s}}$. This quantity is large but finite at $T_{\mathrm{g}}$ and at the thermodynamic glass transition temperature $T_{\mathrm{e}}^{1}$. The configurational entropy is finite too at $T_{\mathrm{e}}^{1}$. It is equal to the residual entropy of glass at $T \rightarrow 0$. Thus, both temperatures $T_{\mathrm{VF}}$ and $T_{\mathrm{K}}$ are fictitious.

The AG ideal glass possesses a single structure state of minimal energy which has no translational invariance and other symmetries. In HPFM, glass is formed by non-crystalline clusters (frozen fluctuons) consisting of $k_{0}$ bids. The configurational entropy of glass is $s_{\mathrm{Sc}}=s_{\mathrm{S}}-s_{\mathrm{cr}} ; s_{\mathrm{cr}}$ is the entropy of crystalline state; $s_{\mathrm{sc}}$ is the residual entropy of glass at $T \rightarrow 0$. 
To determine $T_{\mathrm{K}}$ in HPFM, let us find the liquid entropy,

$$
\begin{aligned}
& s\left(T ; c_{\mathrm{s}}\right)=-\frac{\partial g\left(T ; c_{\mathrm{s}}\right)}{\partial T}=c_{\mathrm{s}} s_{\mathrm{s}}(T)+\left(1-c_{\mathrm{s}}\right) s_{\mathrm{f}}(T)+s_{\text {mix }}\left(c_{\mathrm{s}}\right)=c_{\mathrm{s}} s_{\mathrm{s}}+\Delta s_{\mathrm{f}, \mathrm{s}} c_{\mathrm{f}}+s_{\text {mix }}\left(c_{\mathrm{s}}\right), \\
& s_{\text {mix }}\left(c_{\mathrm{s}}\right)=-\left[c_{\mathrm{s}} \ln c_{\mathrm{s}}+\left(1-c_{\mathrm{s}}\right) \ln \left(1-c_{\mathrm{s}}\right)\right],
\end{aligned}
$$

$s_{\text {mix }}$ with $T \rightarrow T_{\mathrm{e}}^{1}$ is negligibly small and $s_{\mathrm{s}} \rightarrow s_{\mathrm{cr}}+s_{\mathrm{sc}}$. Therefore,

$$
s\left(T ; c_{\mathrm{s}}\right) \approx s_{\mathrm{sc}}+\Delta s_{\mathrm{f}, \mathrm{s}} c_{\mathrm{f}} \approx s_{\mathrm{C}}+\Delta s_{\mathrm{f}, \mathrm{s}}(1-\alpha) .
$$

As it follows from equation (16), in linear on $T-T_{\mathrm{e}}$ approximation

$$
\alpha=\frac{h_{\mathrm{s}, \mathrm{f}}(T)}{2\left(2 T-\tilde{g}_{\mathrm{s}, \mathrm{f}}\right)} \approx \frac{\Delta s_{\mathrm{s}, \mathrm{f}}\left(T_{\mathrm{e}}-T\right)}{2\left(2 T_{\mathrm{e}}-\tilde{g}_{\mathrm{s}, \mathrm{f}}\right)} .
$$

Since at the $T_{\mathrm{K}}$ extrapolated value of $s\left(T ; c_{\mathrm{s}}\right)$ is zero, we have

$$
\alpha\left(T_{\mathrm{K}}\right)=1+\frac{s_{\mathrm{sc}}}{\Delta s_{\mathrm{f}, \mathrm{s}}}
$$

or

$$
T_{\mathrm{K}}=T_{\mathrm{e}}-\frac{2\left(2 T_{\mathrm{e}}-\tilde{g}_{\mathrm{s}, \mathrm{f}}\right)}{\Delta s_{\mathrm{f}, \mathrm{s}}}\left(1+\frac{s_{\mathrm{sc}}}{\Delta s_{\mathrm{f}, \mathrm{s}}}\right) .
$$

Calorimetric data for liquids and glasses show that usually $\Delta s_{\mathrm{f}, \mathrm{s}} \gg s_{\mathrm{sc}}$. Therefore,

$$
T_{\mathrm{K}} \approx T_{\mathrm{e}}-\frac{2\left(2 T_{\mathrm{e}}-\tilde{g}_{\mathrm{s}, \mathrm{f}}\right)}{\Delta s_{\mathrm{f}, \mathrm{s}}}
$$

and $\alpha\left(T_{\mathrm{K}}\right) \approx 1$.

The empiric three parameter Vogel-Fulcher (VF) formula reads

$$
\ln \Gamma_{\alpha}=A+\frac{B}{T-T_{\mathrm{VF}}} .
$$

Here $A, B$ and $T_{\mathrm{VF}}$ are fitting parameters.

To determine $T_{\mathrm{VF}}$ in the HPFM, let us use the method proposed by Stickel at al. [24, 25]. This method of experimental data presentation is known as the Stickel plot.

Noting that the quantity

$$
\gamma^{-1}=\frac{\mathrm{d} \ln \Gamma_{\alpha}}{\mathrm{d} T}=-\frac{B}{\left(T-T_{\mathrm{VF}}\right)^{2}}
$$

depends on two parameters, B and $T_{\mathrm{VF}}$, and that $\gamma=0$ at $T=T_{\mathrm{VF}}$, Stickel has used an extensive set of experimental data on relaxation in supercooled liquids using $\gamma$ to determine both parameters 25].

It follows from (16), (24) and (38) that below $T_{\mathrm{e}}$

$$
\gamma^{-1}=\frac{\mathrm{d} \ln \Gamma_{\alpha}}{\mathrm{d} T}=-\frac{B(\alpha)}{T^{2}}, \quad B(\alpha)=E-\tilde{g}_{\mathrm{sf}} / 2+\frac{z_{\mathrm{C}}}{2}\left(\Delta s_{\mathrm{f}, \mathrm{s}} T_{\mathrm{e}}+z_{\mathrm{C}}^{-1 / 3} g_{\mathrm{sf}}\right)(1+2 \alpha) .
$$

Noting that

$$
\gamma^{1 / 2}=\frac{T}{[B(\alpha)]^{1 / 2}}=\frac{T}{[B(0)(1+2 K \alpha)]^{1 / 2}} \approx \frac{T(1-K \alpha)}{[B(0)]^{1 / 2}}, \quad K=1-\frac{E-\tilde{g}_{\mathrm{sf}} / 2}{B(0)},
$$

we see that the value of $\gamma$ extrapolated to temperature below $T_{\mathrm{e}}$ goes to zero at

$$
\alpha\left(T_{\mathrm{VF}}\right)=\frac{1}{K}=1+\frac{E-\tilde{g}_{\mathrm{sf}} / 2}{B(0)} .
$$


The last term in brackets of r.h.s in this equation is $\approx \ln \left(\Gamma_{\alpha}\left(T_{\mathrm{e}}\right) / \Gamma_{\alpha}\left(T_{\mathrm{e}}^{0}\right)\right) \ll 1$. Therefore,

$$
\alpha\left(T_{\mathrm{VF}}\right) \approx 1
$$

As it follows from (41),

$$
T_{\mathrm{VF}}=T_{\mathrm{e}}-\frac{2\left(2 T_{\mathrm{e}}-\tilde{g}_{\mathrm{s}, \mathrm{f}}\right)}{\Delta s_{\mathrm{f}, \mathrm{s}}}\left(1+\frac{E-\tilde{g}_{\mathrm{sf}} / 2}{B(0)}\right) \approx T_{\mathrm{e}}-\frac{2\left(2 T_{\mathrm{e}}-\tilde{g}_{\mathrm{s}, \mathrm{f}}\right)}{\Delta s_{\mathrm{f}, \mathrm{s}}} .
$$

Comparing (34), (35) and (42), (43) we see that

$$
T_{\mathrm{VF}} \approx T_{\mathrm{K}}
$$

To find correlation of $T_{\mathrm{MCT}}, T_{\mathrm{g}}$ and relation $T_{\mathrm{VF}}$, it has to be noted that $T_{\mathrm{MCT}}$ is close to $T_{\mathrm{e}}$ as Stickel et al. have found [24, 25]. Though $T_{\mathrm{g}}$ is not a properly defined temperature, evidently $c_{\mathrm{s}}\left(T_{\mathrm{g}}\right) \approx 1$ or $\alpha\left(T_{\mathrm{g}}\right) \approx 1 / 2$. Thus, we have from (33) and (42) that

$$
T_{\mathrm{MCT}}-T_{\mathrm{VF}} \approx 2\left(T_{\mathrm{g}}-T_{\mathrm{VF}}\right)
$$

It follows from (23) and (38) that at $T \leqslant T_{\mathrm{e}}^{1}$

$$
\Gamma_{\alpha}(T) \sim \exp \left(-\frac{E+z_{\mathrm{C}} \Delta s_{\mathrm{f}, \mathrm{s}}+z_{\mathrm{C}}^{2 / 3} g_{\mathrm{sf}}}{2 T}\right)
$$

and, consequently,

$$
B=E+z_{\mathrm{C}} \Delta s_{\mathrm{f}, \mathrm{s}}+z_{\mathrm{C}}^{2 / 3} g_{\mathrm{sf}}, \quad T_{\mathrm{VF}}=0 .
$$

Similarly, from (26), (38) we have that at $T \geqslant T_{\mathrm{e}}^{0}$

$$
B=E, \quad T_{\mathrm{VF}}=0 .
$$

Thus, beyond the glass transition temperature range $\left[T_{\mathrm{e}}^{1}, T_{\mathrm{e}}^{0}\right]$ the relaxation rate obeys the Arrhenius equations with different activation energies.

\section{Conclusions}

Taking account for the frustration and volumetric interaction of the solid-like fluctuons in the HPFM leads to renormalization of thermodynamic coefficients saving the mathematical form of the equilibrium state equations. For this reason, equations (3), (7) can be taken as a minimal model of the HPS thermodynamics. The liquid-to-glass transition takes place within the temperature range of width scaled by $\left(g_{\mathrm{ss}}-\varphi_{0}\right)$.

Cooperatively rearranging domains are formed due to the interactions of fluctuons and the rearrangements are thermally activated events. The fact that the activation energy increases and liquid entropy decreases with the temperature decrease lies in the base of the assumption on the non-analytic behavior of entropy and singularity of the $\alpha$-relaxation time at finite temperatures $T_{\mathrm{K}}$ and $T_{\mathrm{VF}}$ which in the AG model coincide but are not accessible due to the violation of ergodicity at $T_{\mathrm{g}}$. In our model, the free energy derivatives have no singularities at $T_{\mathrm{K}}$ and $T_{\mathrm{VF}}$ but these temperature are determined by an extrapolation procedure. As a result, it is found that $T_{\mathrm{VF}} \approx T_{\mathrm{K}}$ and $T_{\mathrm{MCT}}-T_{\mathrm{VF}} \approx 2\left(T_{\mathrm{g}}-T_{\mathrm{VF}}\right)$.

Above $T_{\mathrm{e}}^{0}$ and below $T_{\mathrm{g}}$ the relaxation rate obeys the Arrhenius equations with different activation energies. 


\section{References}

1. Bakai A.S., Low Temp. Phys., 1996, 22(8), 733; Bakai A.S., Low Temp. Phys., 1998, 24(1), 3.

2. Fischer E.W., Bakai A.S., Slow Dynamics in Complex Systems. - In: Proc. AIP Conf. Vol. 469, ed. M. Tokuyama, I. Oppenheim, 1999, p. 325.

3. Fischer E.W., Bakai A., Patkowski A., Steffen W., Reinhardt L., J. Non-Cryst. Solids, 2002, 307-310, 584 .

4. Bakai A.S., Fischer E.W., J. Chem. Phys., 2004, 120, 5235-5252.

5. Bakai A.S., J. Chem. Phys., 2006, 125, 064503.

6. Bakai A.S. - In: Problems of Theoretical Physics, N.F. Shul'ga (Ed.). Kharkov, 2008.

7. Vogel H., Phys. Z., 1921, 22, 645.

8. Fulcher G.S., J. Am. Ceram. Soc., 1925, 8, 329.

9. Kauzmann W., Chem. Rev., 1948, 43, 219.

10. Götze W., Z. Phys. B Con. Mat., 1985, 60, 195.

11. Götze W. - In: Proc. Les Houches Summer School, Course L.I., Hansen J.P., Levesque D., Zinn-Justin J. (Eds.). Elsevier, New-York, 1991, p. 287.

12. Gibbs J.H., Di Marzio E.A., J. Chem. Phys., 1958, 28, 373.

13. Adam G., Gibbs J.H., J. Chem. Phys., 1965, 43, 139.

14. Odagaki T., Phys. Rev. Lett., 1995, 75, 3701.

15. Mohanty U., Diezemann G., Fourkas J.T., J. Chem. Phys., 1995, 113, 3719.

16. Angell C.A., Res. Natl. Inst. Stand. Technol., 1997, 102, 171.

17. Wang L.M., Angell C.A., J. Chem. Phys., 2003, 118, 10353.

18. Frenkel J., J. Chem. Phys., 1939, 7, 538.

19. Cicerone M.T., Blackburn F.R., Ediger M.D., J. Chem. Phys., 1995, 102, 471.

20. Cicerone M.T., Ediger M.D., J. Chem. Phys., 1996, 103, 5684.

21. Silescu H., J. Non-Cryst. Solids, 1999, 243, 81.

22. Ediger M.D., Ann. Rev. Phys. Chem., 2000, 51, 99.

23. Richert R., J. Phys.: Condens. Matter, 2002, 14, R703.

24. Stickel F., Fischer E.W., Richert R., J. Chem. Phys., 1995, 102, 6251.

25. Stickel F. Dissertation, Mainz, 1995.

\section{Термодинаміка і кооперативна динаміка гетерофазної рідини}

\section{О.С.Бакай}

Національний науковий центр “Харківський фізико-технічний інститут” НАН України, вул. Академічна, 1, 61108, Харків, Україна

Розглянуто термодинаміку і кооперативну динаміку гетерофазної рідини з урахуванням фрустрації і об'ємної взаємодії твердотільних флуктуонів. Встановлено, що ширина температурного інтервалу перетворення рідини в скло є пропорційною різниці параметру фрустрації і енергії об'ємної взаємодії. Знайдено уявні температури Кауцмана і Фогеля-Фулчера. Вони виявились близькими з гарною точністю. Розглянуто також кореляцію температури склування, Фогеля-Фулчера і температури “ідеального" перетворення рідина-скло, яке описано в моделі взаємодіючих мод.

Ключові слова: гетерофазні рідкі стани, перехід склування, температури Кауцманна та Фогеля-Фулчера 
